\title{
INTERTWINING MAPS FOR THE WEITZENBÖCK AND CHEBYSHEV DERIVATIONS
}

\author{
L. Bedratyuk, N. Lunio. Intertwining maps for the Weitzenböck and Chebyshev derivations, \\ Mat. Stud. 49 (2018), 3-12. \\ The notions of Chebyshev derivations of the first and the second kind are presented. Explicit \\ forms of the corresponding intertwining maps are found.
}

1. Introduction. Let us consider a family of polynomials $\left\{P_{n}(x)\right\}$, deg $P_{n}(x)=n$ of one variable with rational coefficients. We are interested in finding some polynomial identities for these polynomials, i.e., the identities of the form

$$
F\left(P_{0}(x), P_{1}(x), \ldots, P_{n}(x)\right)=0
$$

where $F\left(x_{0}, x_{1}, \ldots, x_{n}\right)$ is a rational polynomial of $n+1$ variables. It is proved (see, for example, [1]) that the Bernoulli polynomials $B_{n}(x)$ satisfy the following identities

$$
\sum_{i=0}^{n}(-1)^{i}\left(\begin{array}{l}
n \\
i
\end{array}\right) B_{i}(x) B_{n-i}(x)+(n-1) B_{n}=0, \quad n \in \mathbb{N} .
$$

Thus, in this case we have

$$
F\left(x_{0}, x_{1}, \ldots, x_{n}\right)=\sum_{i=0}^{n}(-1)^{i}\left(\begin{array}{l}
n \\
i
\end{array}\right) x_{i} x_{n-i}+(n-1) B_{n},
$$

where $B_{n}$ is the Bernoulli number.

In this paper, we propose a general way of finding such polynomial identities for an arbitrary family $\left\{P_{n}(x)\right\}$ of polynomials.

The basic idea of this method is an application of already known polynomial identities which were found by the first author in [1] using methods of the classical invariant theory and the theory of locally nilpotent derivations to the Appel polynomials.

Let us consider a polynomial sequence $\left\{A_{n}(x)\right\}$, where for all $A_{n}(x)$ the following condition takes place $A_{n}^{\prime}(x)=n A_{n-1}(x), n=0,1,2, \ldots$

The polynomials which possess the property are called the Appel type polynomials, see [2]. In particular, Bernoulli, Euler and Hermite polynomials are the Appel type polynomials.

2010 Mathematics Subject Classification: 05A15, 17B50.

Keywords: Chebyshev derivation; intertwining map; Appel polynomial; recurrence equation; Weitzenböck derivation.

doi:10.15330/ms.49.1.3-12

(C) L. Bedratyuk, N. Lunio, 2018 
The locally nilpotent differentiation of algebra of the polynomials $\mathbb{Q}\left[x_{0}, x_{1}, \ldots, x_{n}\right]$ acts under the following rule $\mathcal{D}_{A}\left(x_{i}\right)=n x_{i-1}$ is closely connected to the Appel polynomials. The kernel of this derivation is called the subalgebra $\operatorname{ker} \mathcal{D}_{A} \subset \mathbb{Q}\left[x_{0}, x_{1}, \ldots, x_{n}\right]$ such that $\mathcal{D}_{A}(f)=0$ for all polynomials $f \in \operatorname{ker} \mathcal{D}_{A}$.

The derivation $\mathcal{D}_{A}$ is so called basis Weitzenböck derivation. The kernel of Weitzenböck derivation is well-studied, it is isomorphic to $S L_{2}$-invariant algebra, see [3], [4]. As it was shown in [1], for every element of $\mathcal{D}_{A}$ there exists some polynomial identity for the Appel polynomials. A sequence of polynomials $\left\{P_{n}(x)\right\}$ can be associated with a derivation $\mathcal{D}_{P}$ of algebra $\mathbb{Q}\left[x_{0}, x_{1}, \ldots, x_{n}\right]$ which is defined as following

$$
\mathcal{D}_{P}\left(x_{i}\right)=\alpha_{i, 0} x_{0}+\alpha_{i, 1} x_{1}+\cdots+\alpha_{i, i-1} x_{i-1}, i=1, \ldots, n,
$$

where the numbers $\alpha_{i, j}$ are found from the conditions

$$
\frac{d}{d x} P_{i}(x)=\alpha_{i, 0} P_{0}(x)+\alpha_{i, 1} P_{1}(x)+\cdots+\alpha_{i, i-1} P_{i-1}(x), i=1, \ldots, n .
$$

Since the polynomial sequence $\left\{P_{n}(x)\right\}$, deg $P_{n}(x)=n$ forms a basis in the vector space of the polynomials of one variable, the numbers $\alpha_{i, j}$ are well-defined.

Let us remark that in the vector space $\left\langle x_{0}, x_{1}, \ldots, x_{n}\right\rangle$ derivations $\mathcal{D}_{P}$ and $\mathcal{D}_{A}$ act as common linear nilpotent operators. Moreover, Jordan normal form of the matrix of the operator $\mathcal{D}_{A}$ consists of a single Jordan block with zero main diagonal. In this case, if the Jordan normal form of the matrix of the operator $\mathcal{D}_{A}$ is formed with a single Jordan block, then there exists a basis in which the action of the matrix of the operator $\mathcal{D}_{P}$ is the same as the action of operator $\mathcal{D}_{A}$.

Thus, there exists an isomorphism $\varphi_{A P}$ from the vector space $\left\langle x_{0}, x_{1}, \ldots, x_{n}\right\rangle$ to the vector space $\left\langle x_{0}, x_{1}, \ldots, x_{n}\right\rangle$, and it could be extended to the corresponding endomorphism $\varphi_{A P}: \mathbb{Q}\left[x_{0}, x_{1}, \ldots, x_{n}\right] \rightarrow \mathbb{Q}\left[x_{0}, x_{1}, \ldots, x_{n}\right]$. The map $\varphi_{A P}$ is called $\left(\mathcal{D}_{\mathcal{A}}, \mathcal{D}_{\mathcal{P}}\right)$-intertwining map. Any such map induces an isomorphism from $\operatorname{ker} \mathcal{D}_{\mathcal{A}}$ to $\operatorname{ker} \mathcal{D}_{\mathcal{P}}$. Therefore, the main idea of obtaining polynomials identities for a polynomial family $\left\{P_{n}(x)\right\}$ is to find the explicit form of $\left(\mathcal{D}_{\mathcal{A}}, \mathcal{D}_{\mathcal{P}}\right)$-intertwining map. Applying the action of the $\varphi_{A P}$ map to the elements of the kernel $\operatorname{ker} \mathcal{D}_{\mathcal{A}}$, we always obtain the elements of the kernel ker $\mathcal{D}_{\mathcal{P}}$. As a consequence, if the polynomial $F\left(x_{0}, x_{1}, \ldots, x_{n}\right)$ belongs to the kernel ker $\mathcal{D}_{\mathcal{A}}$, then the element

$$
\varphi_{A P}\left(F\left(x_{0}, x_{1}, \ldots, x_{n}\right)\right)=F\left(\varphi_{A P}\left(x_{0}\right), \varphi_{A P}\left(x_{1}\right), \ldots, \varphi_{A P}\left(x_{n}\right)\right)
$$

always belongs to the kernel of the derivation $\mathcal{D}_{\mathcal{P}}$ and this polynomial of one variable

$$
F\left(\varphi_{A P}\left(P_{0}(x)\right), \varphi_{A P}\left(P_{1}(x)\right), \ldots, \varphi_{A P}\left(P_{n}(x)\right)\right),
$$

defines a polynomial identity for the polynomial family $P_{n}(x)$. The same approach was used by the first author in [5], [6] to find new polynomial identities in the case of the Fibonacci, Lucas and Kravchuk polynomials.

The subject of this study is finding out an explicit form of $\left(\mathcal{D}_{\mathcal{A}}, \mathcal{D}_{\mathcal{P}}\right)$-intertwining map for Chebyshev polynomials of the first and the second kind.

2. Intertwining map for the Chebyshev derivation of the first kind. The Chebyshev polynomials of the first kind $T_{n}(x)$ and the Chebyshev polynomials of the second kind $U_{n}(x)$ are defined by the following ordinary generating functions

$$
\mathcal{G}\left(T_{n}(x), t\right)=\frac{1-x t}{1-2 x t+t^{2}}=\sum_{n=0}^{\infty} T_{n}(x) t^{n}, \quad \mathcal{G}\left(U_{n}(x), t\right)=\frac{1}{1-2 x t+t^{2}}=\sum_{n=0}^{\infty} U_{n}(x) t^{n} .
$$


The derivatives of the polynomials can be expressed in the terms of the polynomials as follows:

$$
\begin{gathered}
\frac{d}{d x} T_{n}(x)=n\left(\sum_{k=1}^{n-1}\left(1-(-1)^{k}\right) T_{n-k}(x)+\frac{1-(-1)^{n}}{2} T_{0}(x)\right), \\
\frac{d}{d x} U_{n}(x)=\sum_{k=1}^{n}\left(1-(-1)^{n-k}\right)(n-k+1) U_{n-k}(x)=\sum_{k=0}^{[n / 2]}(n-2 k) U_{n-2 k-1}(x),
\end{gathered}
$$

see $[7],[8]$.

This motivates us to give the following

Definition 1. Derivations of $\mathbb{Q}\left[x_{0}, x_{1}, x_{2}, \ldots, x_{n}\right]$ defined by

$$
\begin{gathered}
D_{\mathcal{T}}\left(x_{0}\right)=0, D_{\mathcal{T}}\left(x_{n}\right)=n\left(\sum_{k=1}^{n-1}\left(1-(-1)^{k}\right) x_{n-k}+\frac{1-(-1)^{n}}{2} x_{0}\right), \\
D_{\mathcal{U}}\left(x_{0}\right)=0, D_{\mathcal{U}}\left(x_{n}\right)=\sum_{k=1}^{n-1}\left(1+(-1)^{n-k+1}\right)(k+1) x_{k},
\end{gathered}
$$

are called the Chebyshev derivation of the first kind and the Chebyshev derivation of the second kind, respectively.

We have

$$
\begin{array}{llrl}
D_{\mathcal{T}}\left(x_{0}\right) & =0, & & D_{\mathcal{U}}\left(x_{0}\right)=0, \\
D_{\mathcal{T}}\left(x_{1}\right) & =x_{0}, & D_{\mathcal{U}}\left(x_{1}\right) & =2 x_{0}, \\
D_{\mathcal{T}}\left(x_{2}\right) & =4 x_{1}, & D_{\mathcal{U}}\left(x_{2}\right) & =4 x_{1}, \\
D_{\mathcal{T}}\left(x_{3}\right) & =6 x_{2}+3 x_{0}, & D_{\mathcal{U}}\left(x_{3}\right) & =2\left(3 x_{2}+x_{0}\right), \\
D_{\mathcal{T}}\left(x_{4}\right) & =8 x_{3}+8 x_{1}, & D_{\mathcal{U}}\left(x_{4}\right) & =2\left(4 x_{3}+2 x_{1}\right), \\
D_{\mathcal{T}}\left(x_{5}\right) & =10 x_{4}+10 x_{2}+5 x_{0}, & D_{\mathcal{U}}\left(x_{5}\right) & =2\left(5 x_{4}+3 x_{2}+x_{0}\right), \\
D_{\mathcal{T}}\left(x_{6}\right) & =12 x_{5}+12 x_{3}+12 x_{1}, & D_{\mathcal{U}}\left(x_{6}\right) & =2\left(6 x_{5}+4 x_{3}+2 x_{1}\right), \\
D_{\mathcal{T}}\left(x_{7}\right) & =14 x_{6}+14 x_{4}+14 x_{2}+7 x_{0}, & D_{\mathcal{U}}\left(x_{7}\right) & =2\left(7 x_{6}+5 x_{4}+3 x_{2}+x_{0}\right), \\
D_{\mathcal{T}}\left(x_{8}\right) & =16 x_{7}+16 x_{5}+16 x_{3}+16 x_{1}, & D_{\mathcal{U}}\left(x_{8}\right) & =2\left(8 x_{7}+6 x_{5}+4 x_{3}+2 x_{1}\right) .
\end{array}
$$

It is obvious that these derivations are triangular and, consequently, locally nilpotent. Also, since $D_{\mathcal{T}}^{n}\left(x_{n}\right)=0, D_{\mathcal{T}}^{n-1}\left(x_{n}\right) \neq 0$, and $D_{\mathcal{U}}^{n}\left(x_{n}\right)=0, D_{\mathcal{U}}^{n-1}\left(x_{n}\right) \neq 0$, the Jordan normal form of the matrices of the operators $D_{\mathcal{T}}$ and $D_{\mathcal{U}}$ consists of a single Jordan block.

Let us find a $\left(\mathcal{D}_{\mathcal{A}}, \mathcal{D}_{\mathcal{T}}\right)$-intertwining map. Define the map $\psi_{A T}$ by

$$
\psi_{A T}\left(x_{n}\right)=\alpha_{n}^{(0)} x_{n}+\alpha_{n}^{(1)} x_{n-2}+\alpha_{n}^{(2)} x_{n-4}+\ldots+\alpha_{n}^{(i)} x_{n-2 i}+\ldots+\alpha_{n}^{\left(\left[\frac{n-1}{2}\right]\right)} x_{n-2\left[\frac{n-1}{2}\right]} .
$$

We have

$$
\begin{gathered}
D_{\mathcal{T}}\left(\psi_{A T}\left(x_{n}\right)\right)=2 n \alpha_{n}^{(0)}\left(x_{n-1}+x_{n-3}+x_{n-5}+x_{n-7}+\ldots\right)+\alpha_{n}^{(1)} 2(n-2)\left(x_{n-3}+x_{n-5}+\right. \\
+\ldots)+\alpha_{n}^{(2)} 2(n-4)\left(x_{n-5}+x_{n-7}+\ldots\right)+\ldots=x_{n-1}\left(2 n \alpha_{n}^{(0)}\right)+ \\
\left.+x_{n-3}\left(\alpha_{n}^{(0)} 2 n+\alpha_{n}^{(1)} 2(n-2)\right)+x_{n-5}\left(\alpha_{n}^{(0)} 2 n+\alpha_{n}^{(1)} 2(n-2)+2(n-4) \alpha_{n}^{(2)}\right)+\cdots\right) .
\end{gathered}
$$

On the other hand,

$$
D_{\mathcal{T}_{1}}\left(\psi_{A T}\left(x_{n}\right)\right)=\psi_{A T}\left(\mathcal{D}_{\mathcal{A}}\left(x_{n}\right)\right)=n \psi_{A T}\left(x_{n-1}\right)=n\left(\alpha_{n-1}^{(0)} x_{n-1}+\alpha_{n-1}^{(1)} x_{n-3}+\alpha_{n-1}^{(2)} x_{n-5}+\ldots\right) .
$$

Thus 
Lemma 1. The sequences $\alpha_{n}^{(0)}, \alpha_{n}^{(1)}, \ldots, \alpha_{n}^{\left(\left[\frac{n-1}{2}\right]\right)}$ satisfy the following system of recurrence equations:

$$
\left\{\begin{array}{l}
\alpha_{n}^{(0)} 2 n=n \alpha_{n-1}^{(0)} \\
\alpha_{n}^{(0)} 2 n+\alpha_{n}^{(1)} 2(n-2)=n \alpha_{n-1}^{(1)}, \\
\alpha_{n}^{(0)} 2 n+\alpha_{n}^{(1)} 2(n-2)+2(n-4) \alpha_{n}^{(2)}=n \alpha_{n-1}^{(2)}, \\
\cdots \cdots \\
\alpha_{n}^{(0)} 2 n+\alpha_{n}^{(1)} 2(n-2)+2(n-4) \alpha_{n}^{(2)}+\cdots+2\left(n-2\left[\frac{n-1}{2}\right]\right) \alpha_{n}^{\left(\left[\frac{n-1}{2}\right]\right)}=n \alpha_{n-1}^{\left(\left[\frac{n-1}{2}\right]\right)} .
\end{array}\right.
$$

After simplifying, we obtain

$$
\left\{\begin{array}{l}
\alpha_{n}^{(0)} 2 n=n \alpha_{n-1}^{(0)}, \alpha_{1}^{(0)}=0 \\
\alpha_{n}^{(1)} 2(n-2)=n\left(\alpha_{n-1}^{(1)}-\alpha_{n-1}^{(0)}\right), \alpha_{2}^{(1)}=0 \\
2(n-4) \alpha_{n}^{(2)}=n\left(\alpha_{n-1}^{(2)}-\alpha_{n-1}^{(1)}\right), \alpha_{4}^{(2)}=0 \\
\cdots \\
2(n-2 i) \alpha_{n}^{(i)}=n\left(\alpha_{n-1}^{(i)}-\alpha_{n-1}^{(i-1)}\right), \alpha_{2 i}^{(i)}=0 \\
\cdots \\
2\left(n-2\left[\frac{n-1}{2}\right]\right) \alpha_{n}^{\left(\left[\frac{n-1}{2}\right]\right)}=n\left(\alpha_{n-1}^{\left(\left[\frac{n-1}{2}\right]\right)}-\alpha_{n-1}^{\left(\left[\frac{n-1}{2}\right]-1\right)}\right)
\end{array}\right.
$$

Let $g_{n}$ be a fixed sequence of polynomials and consider the auxiliary recurrence equation

$$
2(n-a) x_{n}=n\left(x_{n-1}-g_{n-1}\right), x_{a}=0, n \geq a .
$$

Lemma 2. Let

$$
x_{n}=-\frac{n^{\underline{a}}}{2^{n}} \sum_{i=a}^{n-1} \frac{2^{i} g_{i}}{i^{\underline{a}}},
$$

where $n^{\underline{a}}:=n(n-1)(n-2) \cdots(n-(a-1))$. Then the sequence $x_{n}$ is a solution of the auxiliary recurrence equation.

Proof. In fact, suppose that

$$
x_{n}=-\frac{n^{\underline{a}}}{2^{n}} \sum_{i=a}^{n-1} \frac{2^{i} g_{i}}{i^{\underline{a}}} .
$$

Then we have

$$
\begin{gathered}
n\left(x_{n-1}-g_{n-1}\right)=n\left(-\frac{(n-1)^{\underline{a}}}{2^{n-1}} \sum_{i=a}^{n-2} \frac{2^{i} g_{i}}{i^{\underline{a}}}-g_{n-1}\right)= \\
=n\left(-\frac{(n-1)^{\underline{a}}}{2^{n-1}} \sum_{i=a}^{n-2} \frac{2^{i} g_{i}}{i^{\underline{a}}}-\frac{2^{n-1}(n-1)^{\underline{a}} g_{n-1}}{2^{n-1}(n-1)^{\underline{a}}}\right)=\frac{n(n-1)^{\underline{a}}}{2^{n-1}}\left(-\sum_{i=a}^{n-2} \frac{2^{i} g_{i}}{i^{\underline{a}}}-\frac{2^{n-1} g_{n-1}}{(n-1)^{\underline{a}}}\right)= \\
=-\frac{n(n-1)^{\underline{a}}}{2^{n-1}} \sum_{i=a}^{n-1} \frac{2^{i} g_{i}}{i^{\underline{a}}}=-2(n-a) \frac{n^{\underline{a}}}{2^{n}} \sum_{i=a}^{n-1} \frac{2^{i} g_{i}}{i^{\underline{a}}}=2(n-a) x_{n} .
\end{gathered}
$$


In particular,

$$
\alpha_{n}^{(0)}=\frac{1}{2^{n-1}}, \quad \alpha_{n}^{(1)}=-\frac{n(n-2)}{2^{n-1}}, \quad \alpha_{n}^{(2)}=\frac{\left(3 n^{2}-19 n+28\right) n(n-1)}{2^{n+1}} .
$$

To find the general solution of the system let us consider the sequence $\alpha_{n}^{(s)}$ in the basis of the falling powers. Put $\alpha_{n}^{(s)}=\frac{1}{2^{n}}\left(\beta_{0}^{(s)} n^{\underline{s}}+\beta_{1}^{(s)} n^{s+1}+\cdots+\beta_{s}^{(s)} n^{2 s}\right)$,

It is easy to see that $(n-s) n^{\underline{s}}=n^{\underline{s+1}}, n(n-1)^{\underline{s}}=n^{\underline{s+1}}$. Then

$$
\begin{gathered}
2(n-2 s) \alpha_{n}^{(s)}=(n-2 s) \frac{1}{2^{n-1}}\left(\beta_{0}^{(s)} n^{\underline{s}}+\beta_{1}^{(s)} n \frac{s+1}{d}+\cdots+\beta_{s}^{(s)} n^{\underline{2 s}}\right)= \\
\left.=\frac{1}{2^{n-1}}\left(((n-s)-s) \beta_{0}^{(s)} n^{\underline{s}}+((n-(s+1))-(s-1))\right) \beta_{1}^{(s)} n^{\frac{s+1}{2}}+\cdots+(n-2 s) \beta_{s}^{(s)} n^{\underline{2 s}}\right)= \\
=\frac{1}{2^{n-1}}\left(\sum_{i=0}^{s} \beta_{i}^{(s)} n^{\frac{s+i+1}{s-1}}-\sum_{i=0}^{s}(s-i) \beta_{i}^{(s)} n^{\frac{s+i}{}}\right) .
\end{gathered}
$$

On the other hand,

$$
\begin{aligned}
n\left(\alpha_{n-1}^{(s)}-\alpha_{n-1}^{(s-1)}\right) & =\frac{1}{2^{n-1}}\left(\sum_{i=0}^{s} n \beta_{i}^{(s)}(n-1)^{\frac{s+i}{}}-\sum_{i=0}^{s-1} n \beta_{i}^{(s-1)}(n-1)^{\frac{s-1+i}{}}\right)= \\
& =\frac{1}{2^{n-1}}\left(\sum_{i=0}^{s} \beta_{i}^{(s)} n^{\frac{s+i+1}{s-1}}-\sum_{i=0}^{s} \beta_{i}^{(s-1)} n^{\frac{s+i}{}}\right) .
\end{aligned}
$$

After equating the corresponding coefficients, we obtain that $\beta_{i}^{(s)}=\frac{\beta_{i}^{(s-i)}}{s-i}, i=0 \ldots s-1$. The coefficient $\beta_{s}^{(s)}$ is found from the initial condition $a_{2 s}^{(s)}=0$. We have

$$
a_{2 s}^{(s)}=\frac{1}{2^{2 s}}\left(\sum_{i=0}^{s-1} \frac{\beta_{i}^{(s-1)}}{s-i}(2 s)^{\frac{s+i}{2}}+\beta_{s}^{(s)}(2 s) !\right)=0 .
$$

It follows that

$$
\beta_{s}^{(s)}=-\frac{1}{(2 s) !} \sum_{i=0}^{s-1} \frac{\beta_{i}^{(s-1)}}{s-i}(2 s)^{\frac{s+i}{}}=\sum_{i=0}^{s-1} \frac{\beta_{i}^{(s-1)}}{(s-i)(s-i) !}=\sum_{i=0}^{s-1} \frac{\beta_{i}^{(s)}}{(s-i) !} .
$$

Therefore, we get the following recurrence relations for the sequences $\beta_{n}^{(s)}$ :

$$
\beta_{0}^{(s)}=\frac{\beta_{0}^{(s-1)}}{s}, \quad \beta_{1}^{(s)}=\frac{\beta_{1}^{(s-1)}}{s-1}, \quad \beta_{2}^{(s)}=\frac{\beta_{1}^{(s-1)}}{s-2}, \quad \ldots \quad \beta_{s-1}^{(s)}=\beta_{1}^{(s-1)}, \quad \beta_{s}^{(s)}:=b_{s}=\sum_{i=0}^{s-1} \frac{\beta_{i}^{(s)}}{(s-i) !} .
$$

Thus, $\beta_{i}^{(s)}=\frac{1}{(s-i) !} b_{i}$, for $i=0, \ldots, s-1$. Therefore, we get the following recurrence relation for the sequence $b_{s}$ :

$$
\sum_{i=0}^{n} \frac{1}{(n-i) !^{2}} b_{n}=0, n>0
$$


Recall the definition of the Bessel function $J_{\alpha}(x)$ :

$$
J_{\alpha}(z)=\sum_{i=0}^{\infty} \frac{(-1)^{i}}{i \Gamma(i+\alpha+1)}\left(\frac{z}{2}\right)^{2 i+\alpha}
$$

Consider the series

$$
\sum_{n=0}^{\infty} \frac{1}{n !^{2}} z^{n}=J_{0}(\sqrt{-4 z}),
$$

and the ordinary generating function $G\left(b_{n}, z\right)=\sum_{n=0}^{\infty} b_{n} x^{n}$. The last relation implies the following one $J_{0}(\sqrt{-4 z}) G\left(b_{n}, z\right)=1$.

Thus, we prove the following statement.

Theorem 1. A Weitzenböck-Chebyshev intertwining map $\psi_{A T}$ of the first kind has the following form

$$
\psi_{A T}\left(x_{n}\right)=x_{n}+\alpha_{n}^{(1)} x_{n-2}+\alpha_{n}^{(2)} x_{n-4}+\ldots+\alpha_{n}^{(i)} x_{n-2 i}+\ldots+\alpha_{n}^{\left(\left[\frac{n-1}{2}\right]\right)} x_{n-2\left[\frac{n-1}{2}\right]},
$$

where

$$
\alpha_{n}^{(s)}=\frac{1}{2^{n}}\left(\frac{1}{s !} b_{0} n^{\underline{s}}+\cdots+\frac{1}{(s-i) !} b_{i} n \frac{s+i}{}+\cdots+b_{s} n \underline{2 s}\right)
$$

and the generating function for $b_{0}, b_{1}, \ldots, b_{n}, \ldots$ is

$$
\sum_{i=0}^{\infty} b_{i} z^{i}=J_{0}^{-1}(\sqrt{-4 z})
$$

3. Intertwining map for the Chebyshev derivation of the second kind. Let us define the linear map $\psi_{A U}$ by

$$
\psi_{A U}\left(x_{n}\right)=\alpha_{n}^{(0)} x_{n}+\alpha_{n}^{(1)} x_{n-2}+\alpha_{n}^{(2)} x_{n-4}+\ldots+\alpha_{n}^{(i)} x_{n-2 i}+\ldots+\alpha_{n}^{\left(\left[\frac{n-1}{2}\right]\right)} x_{n-2\left[\frac{n-1}{2}\right]} .
$$

Let us consider the following statement.

Lemma 3. The sequences $\alpha_{n}^{(0)}, \alpha_{n}^{(1)}, \ldots, \alpha_{n}^{\left(\left[\frac{n-1}{2}\right]\right)}$ satisfy the following system of recurrence equations:

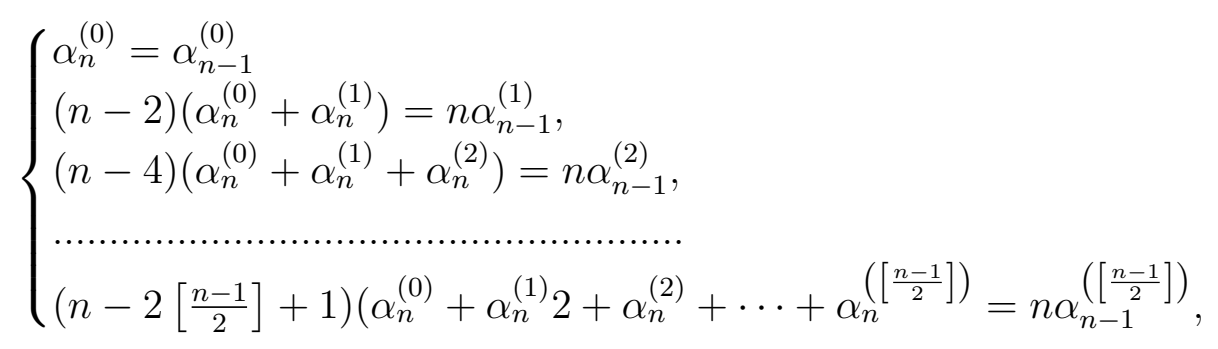


or, equivalently,

$$
\left\{\begin{array}{l}
\alpha_{n}^{(0)}=\alpha_{n-1}^{(0)}, \alpha_{1}^{(0)}=1, \\
\alpha_{n}^{(1)}=\frac{n}{n-2} \alpha_{n-1}^{(1)}-\alpha_{n-1}^{(0)}, \alpha_{2}^{(1)}=0, \\
\alpha_{n}^{(2)}=\frac{n}{n-4} \alpha_{n-1}^{(2)}-\frac{n}{n-2} \alpha_{n-1}^{(1)}, \alpha_{4}^{(2)}=0, \\
\cdots, \\
\alpha_{n}^{(i)}=\frac{n}{n-2 i} \alpha_{n-1}^{(i)}-\frac{n}{n-2(i-1)} \alpha_{n-1}^{(i-1)}, \alpha_{2 i}^{(i)}=0 \\
\ldots, \\
\alpha_{n}^{\left(\left[\frac{n-1}{2}\right]\right)}=\frac{n}{\left.n-2\left[\frac{n-1}{2}\right]\right)} \alpha_{n-1}^{\left(\left[\frac{n-1}{2}\right]\right)}-\frac{n}{\left.n-2\left(\left[\frac{n-1}{2}\right]-1\right)\right)} \alpha_{n-1}^{\left(\left[\frac{n-1}{2}\right]-1\right)} .
\end{array}\right.
$$

The proof is similar to that of Lemma 1.

Let $g_{n}$ be some fixed sequence and consider the auxiliary recurrence equation

$$
x_{n}=n\left(\frac{x_{n-1}}{n-s}-\frac{g_{n-1}}{n-(s-2)}\right), x_{s}=0 .
$$

Lemma 4. The solution of this auxiliary recurrence equation is

$$
x_{n}=-n^{\underline{s}} \sum_{i=s}^{n-1} \frac{g_{i}}{i^{\frac{s-1}{2}}(i-(s-3))} .
$$

Proof. Using the relations

$$
\frac{n(n-1)^{\underline{s}}}{n-1}=n^{\underline{s}}, n(n-1) \frac{s-1}{=} n^{\underline{s}} .
$$

we have

$$
\begin{aligned}
& n\left(\frac{x_{n-1}}{n-s}-\frac{g_{n-1}}{n-(s-2)}\right)=n\left(-\frac{(n-1)^{s}}{n-s} \sum_{i=s}^{n-2} \frac{g_{i}}{i^{\frac{s-1}{2}}(i-(s-3))}-\frac{g_{n-1}}{n-(s-2)}\right)= \\
& =n\left(-\frac{(n-1)^{\frac{s}{s}}}{n-s} \sum_{i=s}^{n-2} \frac{g_{i}}{i^{\frac{s-1}{2}}(i-(s-3))}-\frac{(n-1) \frac{s-1}{m} g_{n-1}}{\left((n-1) \frac{s-1}{2}\right)((n-1)-(s-3))}\right)= \\
& =-\frac{n(n-1)^{\underline{s}}}{n-s} \sum_{i=s}^{n-2} \frac{g_{i}}{i^{\frac{s-1}{2}}(i-(s-3))}-\frac{n(n-1) \frac{s-1}{n} g_{n-1}}{\left((n-1) \frac{s-1}{2}\right)((n-1)-(s-3))}= \\
& =n^{\underline{s}}\left(-\sum_{i=s}^{n-2} \frac{g_{i}}{i^{\frac{s-1}{2}}(i-(s-3))}-\frac{g_{n-1}}{\left((n-1) \frac{s-1}{)}\right)((n-1)-(s-3))}\right)= \\
& =-n^{\underline{s}} \sum_{i=s}^{n-1} \frac{g_{i}}{i^{\frac{s-1}{2}}(i-(s-3))} \text {. }
\end{aligned}
$$

By using the result of Lemma 4, we obtain

$$
\alpha_{n}^{(0)}=1, \alpha_{n}^{(1)}=-\frac{1}{2}(n-1)(n-2), \alpha_{n}^{(2)}=\frac{1}{6}(n-4)(n-3)(n-2) n .
$$


To find a solution for the system consider an unknown sequence $\left\{\alpha_{n}^{(s)}\right\}$ in the basis of the falling powers. Put

$$
\alpha_{n}^{(s)}=(n-(2 s-1))\left(\beta_{0}^{(s)} n \underline{s-1}+\beta_{1}^{(s)} n^{\underline{s}}+\cdots+\beta_{s}^{(s)} n \underline{2 s-1}\right)=(n-(2 s-1)) \sum_{i=0}^{s} \beta_{i}^{(s)} n \frac{s-1+i}{} .
$$

Then we have

$$
\begin{aligned}
& \alpha_{n}^{(s)}=(n-(2 s-1)) \sum_{i=0}^{s} \beta_{i}^{(s)} n \frac{s-1+i}{s-1} \sum_{i=0}^{(s)} \beta_{i}(n-(s+i-1)-(s-i)) n \frac{s+i}{}= \\
& =\sum_{i=0}^{s} \beta_{i}^{(s)}(n-(s+i-1)) n \frac{s+i-1}{s}-\sum_{i=0}^{s} \beta_{i}^{(s)}(s-i) n \frac{s-1+i}{s}=\sum_{i=0}^{s} \beta_{i}^{(s)} n^{\frac{s+i}{s-1}}-\sum_{i=0}^{s}(s-i) \beta_{i}^{(s)} n^{\frac{s-1+i}{}} .
\end{aligned}
$$

On the other hand,

$$
\alpha_{n-1}^{(s)}=(n-2 s) \sum_{i=0}^{s} \beta_{i}^{(s)}(n-1) \frac{s-1+i}{2}, \quad \alpha_{n-1}^{(s-1)}=(n-(2 s-2)) \sum_{i=0}^{s-1} \beta_{i}^{(s-1)}(n-1) \frac{s+i-2}{} .
$$

Consequently,

$$
\begin{gathered}
\alpha_{n}^{(s)}=n\left(\frac{\alpha_{n-1}^{(s)}}{n-2 s}-\frac{\alpha_{n-1}^{(s-1)}}{n-(2 s-2)}\right)=\sum_{i=0}^{s} n \beta_{i}^{(s)}(n-1)^{\frac{s-1+i}{s-1}}-\sum_{i=0}^{s} n \beta_{i}^{(s-1)}(n-1)^{\frac{s-2+i}{}}= \\
=\sum_{i=0}^{s} \beta_{i}^{(s)} n^{\frac{s+i}{s-1}}-\sum_{i=0}^{s} \beta_{i}^{(s-1)} n^{\frac{s-1+i}{2}}
\end{gathered}
$$

Hence,

$$
\sum_{i=0}^{s-1} \beta_{i}^{(s-1)} n^{\frac{s-1+i}{s-1}}=\sum_{i=0}^{s-i) \beta_{i}^{(s)}} n^{\frac{s-1+i}{}} .
$$

By equating the corresponding coefficients of the $n^{\underline{i}}$, we get

$$
\beta_{i}^{(s)}=\frac{\beta_{i}^{(s-1)}}{s-i}, i=0 \ldots s-1 .
$$

The coefficient $\beta_{s}^{(s)}$ is determined from the initial condition $\alpha_{2 s}^{(s)}=0$ that is why

$$
\alpha_{2 s}^{(s)}=\sum_{i=0}^{s} \beta_{i}^{(s)}(2 s)^{\frac{s-1+i}{s-1}}=\sum_{i=0}^{s} \beta_{i}^{(s)}(2 s)^{\frac{s-1+i}{2}}-\beta_{s}^{(s)}(2 s)^{2 s-1}=0 .
$$

Taking into account $(2 s)^{\frac{2 s-1}{2}}=2 s(2 s-1) \ldots 2=(2 s)$ ! we have

$$
\beta_{s}^{(s)}=\frac{1}{(2 s) !} \sum_{i=0}^{s-1} \beta_{i}^{(s)}(2 s)^{\frac{s-1+i}{s-1}}=\sum_{i=0}^{s} \frac{\beta_{i}^{(s)}}{(s-i+1) !} .
$$

We obtain the following relations for $\beta_{n}^{(s)}$ :

$$
\beta_{0}^{(s)}=\frac{\beta_{0}^{(s-1)}}{s}, \beta_{1}^{(s)}=\frac{\beta_{1}^{(s-1)}}{s-1}, \beta_{2}^{(s)}=\frac{\beta_{1}^{(s-1)}}{s-2}, \ldots \beta_{s-1}^{(s)}=\beta_{1}^{(s-1)}, \beta_{s}^{(s)}:=b_{s}=\sum_{i=0}^{s-1} \frac{\beta_{i}^{(s)}}{(s-i+1) !} .
$$


It yields $\beta_{i}^{(s)}=\frac{1}{(s-i) !} b_{i}$, for $i=0, \ldots, s-1$, and

$$
b_{s}=\frac{1}{((s+1) !)^{2}}+\sum_{i=1}^{s-1} \frac{1}{((s-i)(s-i+1) !)} b_{i} .
$$

Therefore, we get the following recurrence relation for the sequence $\left\{b_{i}\right\}$ :

$$
\sum_{i=0}^{s} \frac{1}{(s-i) !(s-i+1) !} b_{i}=0 .
$$

Consider the series

$$
\sum_{n=0}^{\infty} \frac{1}{n !(n+1) !} z^{n}=\frac{1}{\sqrt{z}} J_{1}(\sqrt{-4 z}),
$$

and write

$$
G\left(b_{n}, z\right)=\sum_{n=0}^{\infty} b_{n} z^{n} .
$$

Then, taking into account the recurrence relation we find that

$$
G\left(b_{n}, z\right) \frac{J_{1}(\sqrt{-4 z})}{\sqrt{z}}=1 .
$$

Thus, we have the following statement.

Theorem 2. A Weitzenböck-Chebyshev intertwining map $\psi_{A U}$ of the second kind has the following form

$$
\psi_{A U}\left(x_{n}\right)=x_{n+1}+\alpha_{n}^{(1)} x_{n-1}+\alpha_{n}^{(2)} x_{n-3}+\ldots+\alpha_{n}^{(i)} x_{n+1-2 i}+\ldots+\alpha_{n}^{\left(\left[\frac{n-1}{2}\right]\right)} x_{n+1-2\left[\frac{n-1}{2}\right]},
$$

where

$$
\alpha_{n}^{(s)}=(n-2 s+1)\left(\frac{(-1)^{s}}{s !} b_{0} n \frac{s-1}{2}+\cdots+\frac{(-1)^{s-i}}{(s-i) !} b_{i} n \frac{s+i}{}+\cdots+b_{s} n \frac{2 s-1}{}\right),
$$

and the ordinary generating function for $b_{0}, b_{1}, \ldots, b_{n}, \ldots$ is the following:

$$
\sum_{i=0}^{\infty} b_{i} z^{i}=\frac{\sqrt{z}}{J_{1}(\sqrt{-4 z})} \text {. }
$$

\section{REFERENCES}

1. L. Bedratyuk, Semi-invariants of binary forms and identities for Bernoulli, Euler and Hermite polynomials, Acta Arith., 151 (2012), 361-376.

2. S. Roman, G.-C. Rota, The Umbral Calculus, Advances in Mathematics, 27 (1978), no.2, 95-188.

3. L. Bedratyuk, Kernels of derivations of polynomial rings and Casimir elements, Ukrainian Math. Journal, 62 (2010), no.4, 435-452.

4. L. Bedratyuk, Weitzenböck derivations and the classical invariant theory I, Serdica Math. J., 36 (2010), no.2, 99-120. 
5. L. Bedratyuk, Derivations and Identitites for Fibonacci and Lucas Polynomials, Fibonacci Quart., 51 (2013), no.4, 351-366.

6. L. Bedratyuk, Derivations and identities for Kravchuk polynomials, Ukr. Math. J., 65 (2014), no.12, 1755-1773.

7. L. Fox, I.B. Parker, Chebyshev Polynomials in Numerical Analisys, Oxford Univercity Press, London, 1968.

8. H. Prodinger, Representing derivatives of Chebyshev polynomials by Chebyshev polynomials and related questions, Open Math., 15 (2017), 1156-1160.

Khmelnytsky National University, Khmelnytsk, Ukraine bedratyuk@ief.tup.km.ua 